\title{
Walking reveals trunk orientation bias for visual attention
}

\author{
JEFFERSON D. GRUBB \\ University of Denver, Denver, Colorado \\ and U.S. Navy Aviation Human Systems Integration Laboratory, Monterey, California
}

Catherine L. Reed

University of Denver, Denver, Colorado

and Claremont McKenna College, Claremont, California

AND

\author{
Stefan Bate, John GaRza, AND RaLPh J. Roberts, JR. \\ University of Denver, Denver, Colorado
}

\begin{abstract}
Our trunks influence where we perform actions in space. Thus, trunk direction may define a region of space that is accorded special treatment by the attention system. We investigated conditions under which a trunk orientation bias for attention might be relevant for healthy adults. Three experiments compared visual detection performance for participants standing and walking on a treadmill. Together, the experiments disambiguate the relative contributions of motor activity, motor load, and cognitive load on trunk orientation biases. In Experiment 1, trunk orientation biases (i.e., faster target detection for targets in front of the body midline) were observed in both forward and sideways walking conditions, but not in standing conditions. In Experiment 2, we ruled out the notion that the trunk orientation bias arose from increased motor activity; in fact, the bias was greatest when participants walked at an unusually slow pace. In Experiment 3, we directly compared motor load with cognitive load in a dual-task paradigm; cognitive load influenced overall performance speed, but only motor load produced trunk orientation bias. These results suggest that a trunk orientation bias emerges during walking and motor load conditions.
\end{abstract}

Psychologists have long recognized that people have finite capacities to attend to different sources of information (Broadbent, 1958; Deutsch \& Deutsch, 1963; James, 1948). Because we live in a dynamic environment and cannot fully attend to all information at once, we must flexibly deploy our limited attentional resources in different degrees to various information streams (Kahneman, 1973). Although researchers have identified a number of factors that influence the deployment of attention, until recently the influence of trunk orientation has been largely ignored or taken for granted.

To the extent that the configuration of our body parts constrain, our actions at any moment, they influence where we direct spatial attention. Our perceptual systems serve to plan adaptive actions (Previc, 1998; Prinz, 1997), and attention is the process by which we filter and select the most task-relevant perceptual information (Kahneman \& Treisman, 1984). Consequently, attention should be deployed to those regions of space most relevant for performing the action.

In particular, trunk orientation should be an important anchor for spatial attention (Jeannerod \& Biguer, 1987; Karnath, Schenkel, \& Fischer, 1991; Ventre, Flandrin,
\& Jeannerod, 1984). When using sensory information to guide actions, we must determine the relative configuration of the sensory and effector organs. For example, a visually detected object's retinotopic position indicates its location relative to the eye, but we need to know the object's position relative to the hand in order to plan an appropriate reach. This sensorimotor transformation requires that we account for the orientation of each connected structure between the eye and hand-orientation of the eye relative to the head, orientation of the lower arm relative to the upper arm, and so on (Biguer, Jeannerod, \& Prablanc, 1985; Karnath, 1997). Structurally, the trunk is the hub to which our head, arms, and legs are flexibly attached. As a result, trunk orientation influences sensorimotor planning for many typical actions and should therefore affect the distribution of spatial attention.

The trunk is often aligned with behaviorally important regions of space. First, although we may turn our head and eyes to look in other directions, we usually move in the direction in which the trunk points. The direction of attention toward the path of motion helps us to avoid collisions when locomoting through the environment. A trunk orientation bias for attention would literally help

C. L. Reed, cathy.reed@cmc.edu 
us to watch where we are going. Second, it is convenient to align the trunk with objects that we intend to manipulate. The external space immediately in front of the trunk can be easily reached with one or both hands. Third, the neck is in its neutral position biomechanically when the head midline is aligned with the trunk midline. Maintaining this neutral position requires less energy and is much more comfortable than maintaining a posture in which the head and trunk are not aligned (Bridger, 1995). Indeed, many ergonomics and workplace safety guidelines specifically recommend designing workstations to avoid repeated or maintained head rotations (e.g., National Aeronautics and Space Administration Glenn Research Center, 2002; Oregon Occupational Safety and Health Administration, 2002). Assuming that people tend to do what is naturally most convenient, comfortable, and effective, people should align their trunks with the focus of long-term attention (i.e., their primary interest), especially when locomoting through the environment, and turn their heads only to temporarily focus on secondary interests. As such, trunk orientation is a marker for behaviorally important regions of space. An attentional bias toward such a region would alert us to sudden events occurring there even as we temporarily look elsewhere. In this paper, we will refer to this trunk-defined attentional bias as the trunk orientation hypothesis.

To date, most evidence indicating the existence of a trunk orientation bias for attention comes from studies of patients with unilateral neglect. Following brain injury, typically to the right inferior parietal region, patients with neglect fail to attend to and explore contralesional space (e.g., Bradshaw \& Mattingley, 1995; Brain, 1941). Patients with neglect are better able to explore and to detect targets in contralesional space when their torsos are rotated toward contralesional space (Karnath, Christ, \& Hartje, 1993; Karnath et al., 1991). Additionally, patients' symptoms improve during procedures that induce a displacement of the perceived orientation of the body midline toward the contralesional side. These procedures include cold caloric irrigation of the contralesional ear and warm caloric irrigation of the ipsilesional ear (Rubens, 1985), vibration of the contralesional posterior neck muscles (Karnath, 1994), and viewing of a contralesionally moving optokinetic display (Pizzamiglio, Frasca, Guariglia, Incoccia, \& Antonucci, 1990). These same procedures affect the perception of body midline in neurologically intact participants (Vallar, Guariglia, \& Rusconi, 1997). In sum, patients' symptoms improve when the actual or perceived orientation of their trunk is rotated toward the neglected region of space. Thus, attention appears to follow perceived trunk orientation for patients with neglect.

However, similar findings have been less reliably demonstrated in other brain-injured or neurologically intact populations. Although Karnath and colleagues found that actual trunk orientation affected neglect patients' saccadic response times (Karnath et al., 1991) and neglect patients' ability to detect and name contralesional targets (Karnath et al., 1993), they did not find equivalent effects in neurologically intact or even in brain-injured controls. Rorden,
Karnath, and Driver (2001) found that neck muscle vibration and caloric irrigation reliably shifted healthy participants' perceived trunk orientation, but did not affect participants' performance on simple tasks known to be sensitive to manipulations of spatial attention.

In contrast, other studies of healthy participants have revealed effects of trunk orientation. In a lateralized, targetdetection paradigm, Hasselbach-Heitzeg and ReuterLorenz (2002) found that participants, when their trunks were turned to the right, were slightly faster to respond to targets presented on the right than they were to targets presented on the left. Further, Grubb and Reed (2002) used a covert-orienting paradigm to show that participants exhibited neglect-like effects when their trunks were turned left: They were slightly faster to detect invalidly cued targets on the left and slightly slower to detect invalidly cued targets on the right.

We address the question of what conditions can reliably produce effects of trunk orientation and thereby provide insight into how the trunk may be used for spatial cognition and attention. We hypothesize that one reason for the inconsistent findings in the literature is that the tasks used in previous experiments do not consider factors that may necessitate a trunk orientation bias in everyday life. For example, walking might induce a trunk orientation bias via the introduction of locomotion and additional motor and cognitive processing demands. In studies for which trunk orientation was not found, participants typically performed one relatively simple attention task while sitting still in a static environment. Because participants were not at risk of colliding with anything and were able to devote their undivided attention to the experimental task, any trunk orientation bias may have been actively suppressed, too subtle to compete with participants' focus on the task, or just not required. Following this logic, healthy participants should be more likely to demonstrate trunk orientation effects on tasks that are more motorically and cognitively demanding as well as for which the trunk has greater task relevance.

In this study, we investigated the conditions under which a trunk orientation bias could be reliably demonstrated in healthy individuals. We conducted three experiments to determine whether trunk orientation effects could be enhanced by increasing the demands of the task and by increasing the task-relevance of trunk orientation to the task. In each experiment, we compared lateralized visual detection performance (e.g., Hasselbach-Heitzeg \& ReuterLorenz, 2002) for participants standing and walking on a treadmill. In Experiment 1, to determine whether the act of walking induces a trunk orientation bias and whether any bias is related to the perceived direction of motion, we compare the influence of trunk orientation on detection time under conditions of standing, walking forward, and walking sideways. In Experiment 2, we investigate whether motor activity and arousal per se influence the trunk orientation bias. Finally, in Experiment 3, we contrast the effects of motor load and cognitive load on trunk orientation bias. Together, the experiments disambiguate the relative contributions of motor activity, motor load, and cognitive load on trunk orientation biases. 


\section{EXPERIMENT 1}

In Experiment 1, we investigated whether trunk orientation biases would be observed when healthy participants performed a task in which the trunk would be relevant for spatial attention and cognition-namely, walking. Participants in most attention studies perform experimental tasks while sitting still. Such studies could miss attentional effects related to the demands of locomotion and moving through the environment. For example, when you are walking down a sidewalk, it is the direction of the trunk midline, rather than the direction of the head, that directly influences whether you stay on the sidewalk. To explore whether locomotion enhances the trunk orientation bias on attention, we asked participants to perform a simple target detection task while either standing or walking on a treadmill. We predicted that, overall, participants would demonstrate greater trunk orientation bias (i.e., faster response times [RTs] for targets located in line with the trunk midline) when walking than when standing still.

However, at least two explanations could account for this trunk orientation bias, or enhanced spatial processing. First, when walking, we risk colliding with objects in our path, so it would be beneficial to have a bias to attend in the direction of motion in order to avoid collisions. Because we usually walk in the direction in which the trunk points, such a collision avoidance mechanism would typically amount to a trunk orientation bias for attention. Second, walking represents a secondary task. An enhancement of the trunk orientation effect while walking could merely indicate that the trunk orientation effect is enhanced with increased task demands.

To pit the task demands hypothesis against the collision avoidance hypothesis, we dissociated the direction of simulated motion from trunk orientation by having participants walk both forward and sideways on the treadmill. The task demands hypothesis predicts that participants should show an attentional bias in the direction that the trunk points, regardless of the direction of motion. The collision avoidance hypothesis predicts that participants should show an attentional bias in the direction of simulated motion, regardless of the direction in which the trunk is pointed.

\section{Method}

Participants. Participants were 16 right-handed undergraduates from the University of Denver. Participants had no history of weekly treadmill use and had not exercised on a treadmill for at least 1 month. Each participant gave informed consent and received partial course credit.

Apparatus. The experimental setup is illustrated in Figure 1. The relative trunk and head alignment is consistent with that described in Hasselbach-Heitzeg and Reuter-Lorenz (2002). For all conditions, participants stood or walked on a Smooth 5.0P motorized treadmill (InternetFitness.com). To give participants the sense of moving even though they were not, the room in which they performed the treadmill tasks was darkened, so that the lack of optic flow information was less apparent. In the forward walking condition, body midline corresponded to the movement direction of the tread on the treadmill. In the sideways walking condition, body midline was perpendicular to the movement direction of the tread on the treadmill. In all conditions, the pelvis was perpendicular to the body midline plane.
Participants grasped handrails to maintain proper torso alignment. In the forward walking condition, participants grasped lateral handrails on each side of the treadmill. In the sideways walking condition, participants grasped one handrail with both hands. To ensure proper head alignment, participants placed their chins in a chinrest that maintained head direction at a $45^{\circ}$ angle to the left or right of body midline. (Throughout this paper, trunk orientation refers to the direction of body midline.)

The target detection task was modeled after the tasks described in Hasselbach-Heitzeg and Reuter-Lorenz (2002). The visual stimuli were three light-emitting diodes (LEDs) placed at the ends of two perpendicular rods (i.e., a cross). One red fixation LED was mounted at the far end of the central rod, aligned with the head midline and positioned $57 \mathrm{~cm}$ from the participant's face. Two green target LEDs were mounted at opposite ends of the horizontally oriented bar, also $57 \mathrm{~cm}$ from the participant's face, but at $45^{\circ}$ from the head midline. This setup placed one green target light directly in line with the body midline and the other directly to the side of one shoulder. Auditory cues were presented through a pair of Sony (MDR-AV270) headphones. A computer running E-Prime 1.1 controlled all stimuli and recorded participants' responses.
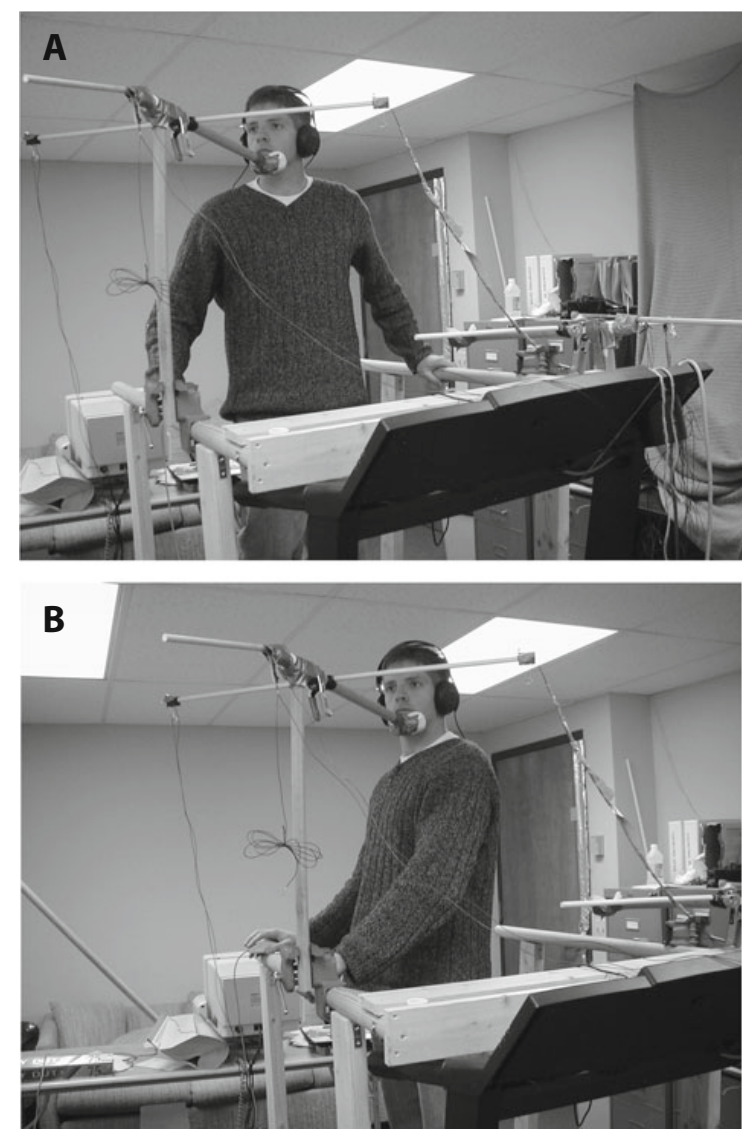

Figure 1. The experimental setup. Participants stood or walked on a treadmill with their heads held with a chinrest at a $45^{\circ}$ angle relative to their trunk midline. Targets appeared to the left and right of fixation. (A) The forward walking condition, in which trunk orientation matched the direction of motion. (B) The sideways walking condition, in which trunk orientation was perpendicular to the direction of motion. 
Procedure. Before each block of trials, participants grasped one handrail with their left hand and a computer mouse, attached to the right handrail, with their right hand. For "standing" blocks, the participants placed their chins on chinrests that were aligned to the left or right. For "walking" blocks, the experimenter first activated the treadmill. When the participants were comfortable with the speed, they placed their chins on the chinrests. For all blocks, the experimenter verified that the participants' heads and trunks were aligned correctly and then extinguished all lights in the room except for the fixation light.

Trials began with a $250-\mathrm{msec}$ cue tone presented over the headphones. For experimental trials, one of the target lights was illuminated for $100 \mathrm{msec}$ after a variable ISI of $200-500 \mathrm{msec}$. The probability that the left and right targets would illuminate was equal. In each block, $85 \%$ of trials were experimental trials. The remaining $15 \%$ of trials were catch trials, in which no target flash followed the cue tone. For these trials, the participants were instructed not to make any response. There were 80 trials in each block.

In summary, participants were instructed to look directly at the fixation light at all times throughout each block of trials. In all conditions (i.e., walking or standing), they were informed that they would hear an auditory cue indicating that the trial would begin and that their task was to detect whether the left or the right green target light appeared. If they saw the light, they were to press the mouse button that corresponded to the target side.

The experiment consisted of one practice block, in which participants stood still, and eight blocks of experimental trials. Half the experimental trials were the forward walking condition: Participants performed the detection task while standing or walking with their trunks aligned with the direction of motion (i.e., toward the front of the treadmill) (see Figure 1A). The other blocks were the sideways walking condition: Participants grasped one handrail with both hands and stood or walked with their trunks perpendicular to the direction of motion of the treadmill (see Figure 1B). For the sideways walking blocks, participants were instructed to shuffle rather than to cross their feet. This kept their trunks in proper alignment and parallel with the handrail. It also prevented them from accidentally stepping off the treadmill's moving surface. The experimental blocks were presented in random order.

Pilot testing revealed that shuffling sideways was more awkward than walking forward at the same speed. Consequently, the treadmill was set to its slowest speed, $0.96 \mathrm{~km} / \mathrm{h}$, for the sideways condition. To equate for effort across walking conditions, the speed of the treadmill was set to $2.41 \mathrm{~km} / \mathrm{h}$ in the aligned condition.

\section{Results and Discussion}

Two participants did not complete the experiment, and their data was not included in the reported analyses. Trials on which participants responded incorrectly, or responded outside a window of 100-2,000 msec after onset of the target, were excluded from further analysis. This filtering resulted in the loss of less than $2 \%$ of trials per block, on average, across participants.

The median RTs from the remaining data were submitted to a repeated measures ANOVA with factors of alignment (2: forward, sideways), motion (2: standing, walking), trunk orientation (2: trunk left, trunk right), and target side (2: target left, target right). In general, participants' RTs were faster for forward than for sideways walking conditions $\left[F(1,13)=11.13, M S_{\mathrm{e}}=9,623.79, p=\right.$ $.005]$. The trend toward a motion $\times$ alignment interaction suggests that this effect was driven by a tendency for participants to respond faster in the forward walking condition $\left[F(1,13)=3.82, M S_{\mathrm{e}}=743,985, p=.06\right]$.

In addition, a trunk orientation $\times$ target side interaction $\left[F(1,13)=5.66, M S_{\mathrm{e}}=1,135.62, p=.03\right]$ was found. Averaged across all alignment and motion conditions, participants were faster to respond to targets that appeared in front of their trunks than to those that appeared to the side of their shoulders. Thus, participants showed a general trunk orientation bias for spatial attention. However, the movement $\times$ trunk orientation $\times$ target side interaction $\left[F(1,13)=6.52, M S_{\mathrm{e}}=1,769.51\right.$, $p=.02$ ] indicates that strength of this trunk orientation bias differed with movement condition. As can be seen in Figure 2, in the two walking conditions participants consistently responded faster to the target in front of their trunks than to the target to the side of their shoulders. It is important to note that this was the case even when participants walked sideways. In contrast to the walking conditions, participants in the standing conditions were generally faster to respond to the left target than to the right target. Consequently, participants only demonstrated a trunk orientation bias for visual attention when walking. No other main effects or interactions were significant.

In summary, the results of Experiment 1 demonstrate that a trunk orientation bias is observed only in walking conditions. The presence of this bias in both forward and sideways walking conditions indicates that it is genuinely related to trunk orientation rather than to the direction of motion. The results highlight the importance of testing conditions in attention studies that might be relevant to everyday activities such as walking. Walking on the treadmill increased participants' tendency to attend to the direction in which the trunk pointed. Nonetheless, this experiment cannot determine whether it is motor activity per se that produces a trunk orientation bias or the increased attentional load produced by walking. We address these questions in Experiments 2 and 3.

\section{EXPERIMENT 2}

In Experiment 2, we determined how walking contributes to the trunk biased processing observed in Experiment 1. Our aim was to determine whether the trunk orientation bias during walking was related directly to increased motor activity or to the combined motor and attention demands of walking more slowly than typical walking speed. Compared with walking at a normal pace $(3.70 \mathrm{~km} / \mathrm{h})$, pilot participants reported that walking at $4.83 \mathrm{~km} / \mathrm{h}$ was more physically demanding but not more attentionally demanding. However, participants in Experiment 1 spontaneously reported that they had to concentrate on walking slowly to match the speed of the treadmill during the walking conditions. The walking speed $(2.41 \mathrm{~km} / \mathrm{h})$ was substantially slower than a typical walking pace $(3.70 \mathrm{~km} / \mathrm{h})$ (Diedrich \& Warren, 1995). Pilot participants reported that walking at $2.41 \mathrm{~km} / \mathrm{h}$ - the speed in the forward walking condition of Experiment 1-was more attention demanding, but less physically demanding, than was walking faster. Thus, trunk orientation biases could be related to increased attention demands associated with walking slowly.

To determine whether the trunk orientation biases observed in Experiment 1 were related more to the increase in motor activity or to attention demands associated with 


\section{Experiment 1}

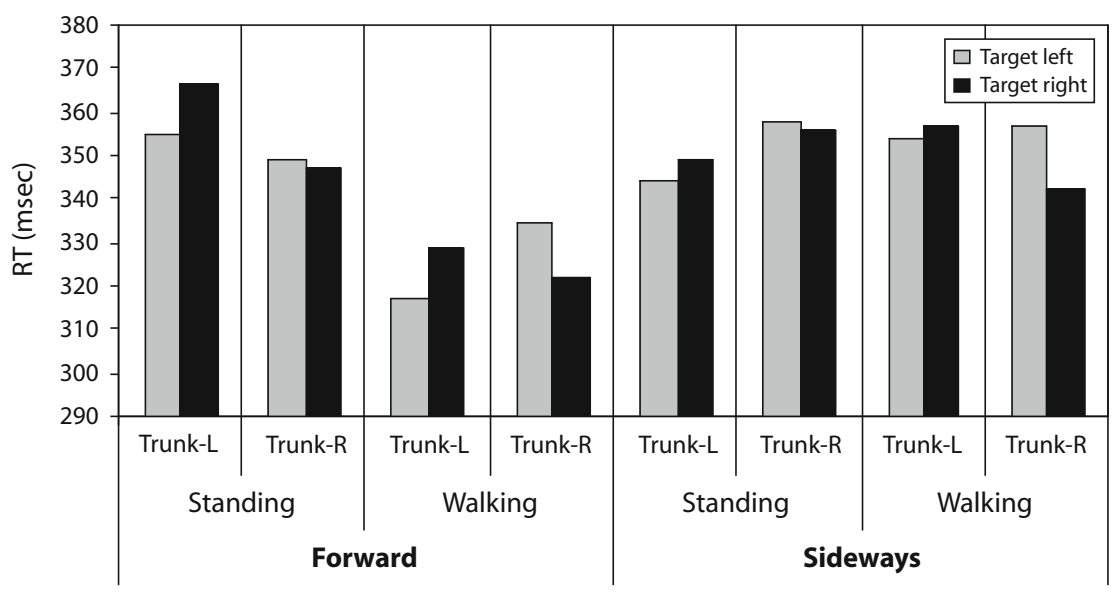

Figure 2. Results of Experiment 1: Trunk orientation $\times$ target side interaction. On the $x$-axis, Trunk-L and Trunk-R refer to the orientation of the trunk midline relative to the head midline. Standing participants responded more quickly to targets on the left. In contrast, walking participants responded more quickly to targets appearing in front of their trunks than to targets appearing off of their shoulders. This trunk orientation bias was observed for both forward and sideways walking conditions, but not for standing conditions.

walking, we compared performance on the same detection task used in Experiment 1 at different walking speeds. If increasing the attention demands of the task enhanced the trunk orientation bias, the bias would be stronger when participants walked at the slow pace. In contrast, if increasing the physical demands of the task enhanced the trunk orientation bias, the bias would be stronger when participants walked at the fast pace.

\section{Method}

Participants. Participants were 15 right-handed students from the University of Denver. Participants had no history of weekly treadmill use and had not exercised on a treadmill for at least 1 month. Participants gave informed consent and received partial course credit.

Apparatus. The same apparatus was used as in Experiment 1.

Procedure. There were four walking speed condition blocks: standing $(0 \mathrm{~km} / \mathrm{h})$; walking at a slow pace $(2.41 \mathrm{~km} / \mathrm{h})$; walking at a normal pace $(3.70 \mathrm{~km} / \mathrm{h})$; and walking at a fast pace $(4.83 \mathrm{~km} / \mathrm{h})$. In each of these blocks, participants faced forward as they stood or walked on the treadmill. These conditions were similar to those in the forward walking conditions of Experiment 1. Otherwise, the detection task and procedure were the same as in Experiment 1.

\section{Results and Discussion}

Trials on which participants responded incorrectly, or outside a window of 100-2,000 msec after onset of the target, were excluded from further analysis. This filtering resulted in the loss of less than $2 \%$ of trials per block, on average, across participants. From the remaining trials, participants' median RTs were calculated.

Participants' median RTs were submitted to a repeated measures ANOVA with factors of trunk orientation (2: trunk left, trunk right), target side (2: left target, right target), and speed (4: $0.0,2.41,3.70,4.83 \mathrm{~km} / \mathrm{h}$ ). Additionally, trunk orientation $\times$ target side planned comparisons were performed to test for trunk orientation effects at each speed.
The Greenhouse-Geisser technique was used to correct for potential violations of sphericity in analyses that contained the four-level speed factor.

In the critical test of the trunk orientation hypothesis, the overall ANOVA revealed a trunk orientation $\times$ target side interaction $\left[F(1,14)=6.87, M S_{\mathrm{e}}=1,039.58, p=.02\right]$. However, the trunk orientation $\times$ target side $\times$ speed interaction $\left[F(3,42)=3.30, M S_{\mathrm{e}}=358.69, p=.04\right]$ indicated that this trunk orientation effect differed depending on how fast the participants walked. Descriptively, when walking at between 2.41 and $4.83 \mathrm{~km} / \mathrm{h}$, participants were faster to respond to the target in front of their trunk than to the target that was off of their shoulder (see Figure 3). This is the predicted pattern of results if participants had an attentional bias for trunk orientation. In contrast, participants were faster to respond to the left target than to the right target when they were standing still $(0 \mathrm{~km} / \mathrm{h}$ condition $)$, regardless of trunk orientation. Moreover, although participants responded fastest to the target in front of the trunk in each of the walking conditions, the magnitude of this bias decreased as speed increased. Planned comparisons revealed that the trunk orientation $\times$ target side interaction was significant when participants walked at $2.41 \mathrm{~km} / \mathrm{h}$ $\left[F(1,14)=13.61, M S_{\mathrm{e}}=1,921.00, p=.002\right]$, but not significant when they walked at $3.70 \mathrm{~km} / \mathrm{h}[F(1,14)=1.45$, $\left.M S_{\mathrm{e}}=163.50, p=.25\right]$ or $4.83 \mathrm{~km} / \mathrm{h}[F(1,14)<1]$.

Additionally, the overall ANOVA also revealed a significant main effect for speed $\left[F(2,37)=4.88, M S_{\mathrm{e}}=\right.$ $2,731.39, p<.01]$. Participants responded more quickly the faster they walked. Such speeded responses could reflect increases in arousal as the pace increased. No other main effects or interactions approached significance.

In summary, the results of Experiment 2 are most consistent with the hypothesis that trunk orientation has an effect on spatial attention when it is relevant to the task 


\section{Experiment 2}

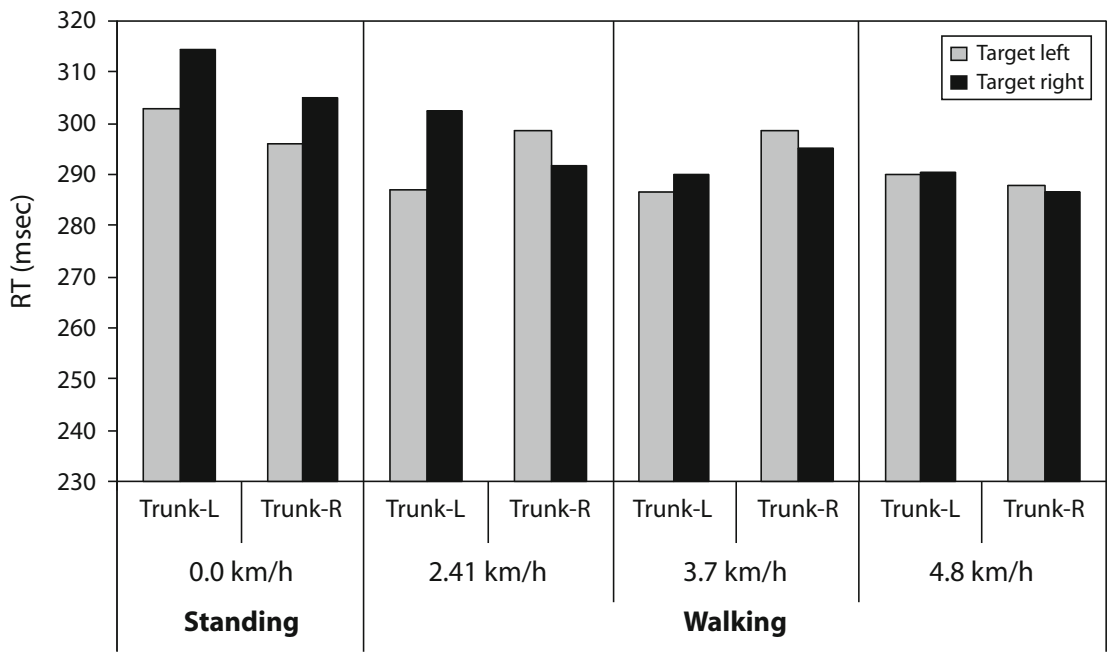

Figure 3. Results of Experiment 2: Trunk orientation $\times$ target side $\times$ walking speed interaction. On the $x$-axis, Trunk-L and Trunk-R refer to the orientation of the trunk midline relative to the head midline. When standing still, participants responded more quickly to targets on the left. When walking, participants responded more quickly to targets appearing in front of their trunk. This trunk orientation bias was greatest for the $2.41 \mathrm{~km} / \mathrm{h}$ walking speed, which is abnormally slow for most people.

and when the task has attention demands. The trunk orientation bias was found only during walking conditions and not during standing conditions; this replicates the results of Experiment 1. Further, the bias was related to attention demands rather than to increased motor activity demands: The bias was only significant for the walking speed requiring the greatest amount of concentration, not the fastest walking speed. Faster walking speeds had an effect in that they decreased overall RTs, suggesting greater levels of general arousal.

Experiment 2 demonstrated that attentional load influences the emergence of a trunk orientation bias. Nonetheless, this experiment was not designed to determine whether the attentional demands came from motor or cognitive origins. In Experiment 3, we address this issue directly.

\section{EXPERIMENT 3}

Although the trunk orientation bias was strongest at the slowest walking speed, Experiment 2 could not determine whether the "extra concentration" required to walk at the slow pace derived from a greater motor load, a greater cognitive load, or both. In other words, because both motor and cognitive loads were present in the slow walking condition of Experiment 2, motor load and/or cognitive load could influence the trunk orientation effect. In order to test this issue, we conducted a third experiment that directly compared motor load with cognitive load, using a dual-task paradigm. As in previous experiments, we assessed motor load by including walking and standing conditions. We induced a purely cognitive, nonmotoric load by comparing performance with and without a concurrent auditory counting task. Attending to the tones, and maintaining the number presented in working memory, has been shown to load generally on cognitive processing resources (e.g., Jimenez \& Vazquez, 2005).

Specifically, we used a $2 \times 2$ factorial design in which participants performed the same visual target detection from previous experiments while either standing or walking on the treadmill, and either counting or not counting the tones. If the trunk orientation effect is found only in the walking conditions and is not affected by cognitive load, the bias can be attributed to motor activity. Alternatively, if the effect is found during the counting task but is not affected by motor load, the bias can be attributed primarily to cognitive load. However, there may also be an interaction between motor and cognitive loads, which would be indicated by an effect only when participants perform both the walking and the counting tasks, but not when the tasks are performed independently.

\section{Method}

Participants. Participants were 16 right-handed undergraduates from the University of Denver. Participants had no history of weekly treadmill use and had not exercised on a treadmill for at least one month. Each participant gave informed consent and received partial course credit.

Apparatus. The apparatus was the same as that used in Experiments 1 and 2 .

Procedure. There were four condition blocks: (1) standing-no counting; (2) standing-counting; (3) walking-no counting; and (4) walking-counting. In each of these blocks, participants faced forward as they stood or walked on the treadmill. The procedure was the same as that described in Experiment 1, except that the cue tone at the beginning of each trial was either high $(800 \mathrm{~Hz})$ or low $(400 \mathrm{~Hz})$ and lasted $200 \mathrm{msec}$. The two tones were presented randomly with replacement, so that every participant and block had a unique, unpredictable number of high versus low tones. Prior to 
Experiment 3

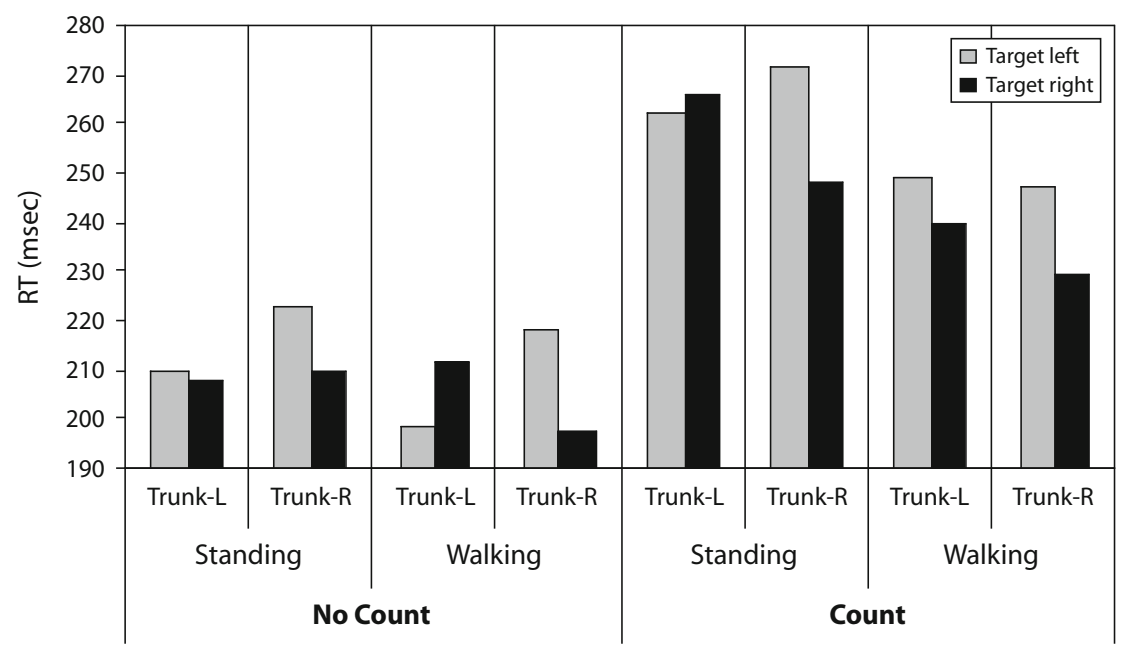

Figure 4. Results of Experiment 3: Trunk orientation $\times$ target side $\times$ movement $\times$ counting interaction. On the $x$-axis, Trunk-L and Trunk-R refer to the orientation of the trunk midline relative to the head midline. The trunk orientation bias was observed only for the walking-no counting condition, indicating a contribution of motor load to trunk orientation bias. Although cognitive load produced an overall slowing of response time, it did not produce a trunk orientation bias, nor did it interact with motor load manipulations.

experimental trials, participants were given practice identifying high and low tones to ensure that they could discriminate between them. For the counting conditions, participants were required to count how many high tones they heard throughout the trials and report that number to the experimenter at the end of the block.

\section{Results and Discussion}

As in the previous experiments, trials on which participants responded incorrectly or outside a window of $100-2,000$ msec after onset of the target were excluded from further analysis. As in the previous experiments, this filtering resulted in the loss of less than $2 \%$ of trials per block, on average, across participants. One participant was eliminated from the analyses because his RTs were 3 standard deviations ( $S D \mathrm{~s})$ slower than the overall participants' mean. From the remaining trials, participants' median RTs were calculated. The mean error rate across participants for counting tones in a particular block was $2.41 \%(S D=1.5 \%)$.

Participants' median RTs were submitted to a repeated measures ANOVA with factors of movement (2: standing, walking), counting (2: no count, count), trunk orientation (2: trunk left, trunk right), and target side (2: left target, right target). A main effect of movement was found $[F(1,14)=$ $\left.7.21, M S_{\mathrm{e}}=1,476.68, p<.02\right]$ in which participants responded more quickly when walking than standing, suggesting an influence of arousal. A significant main effect of counting was found $\left[F(1,14)=24.13, M S_{\mathrm{e}}=4,456.20\right.$, $p<.0001]$ in which counting RTs were slower overall, indicating that participants were performing the dual task. Of interest, the interaction between movement and counting was not significant $(F<1$, n.s.), verifying that the two tasks loaded independently on motor and cognitive systems, respectively. Importantly, the significant trunk orientation $\times$ target side interaction was replicated from the previous experiments $\left[F(1,14)=10.87, M S_{\mathrm{e}}=555.16\right.$, $p<.01]$, but was mediated by the four-way interaction $\left[F(1,14)=5.38, M S_{\mathrm{e}}=322.45, p<.05\right]$. The interactions revealed a trunk orientation bias in the walking-no counting condition only $\left[F(1,14)=32.80, M S_{\mathrm{e}}=131.13, p<\right.$ $.001]$. No other conditions produced a trunk orientation bias (all $p \mathrm{~s}>.05$ ), and no other main effects or interactions approached significance (all $p \mathrm{~s}>.05$ ).

In sum, Experiment 3 demonstrated that trunk orientation biases appear to be more strongly related to motor load than to cognitive load. Motor load by itself produced relatively faster RTs in the direction of the trunk than toward the head; in addition, motor activity generally speeded RTs. Influences of cognitive load were apparent in that it generally slowed RTs but did not interact with trunk orientation. Further, any interactions between motor and cognitive load were not related to trunk orientation bias.

\section{GENERAL DISCUSSION}

In this study, we investigated whether trunk direction biases attention and the conditions under which it could be revealed. Our focus on potential contributions of the trunk to spatial cognition comes from consideration of ecological conditions, in which the trunk might be important for performing actions. For example, we tend to align our trunks with important regions of space. It is easiest to manipulate and work on items when they are directly in front of the trunk. Also, it is easiest to move in the direction in which the trunk points. We tend to point our trunks toward objects and directions of interest. At the same time, 
our head and eyes are free to turn and look in other directions, even though they too are mostly aligned with the trunk midline. Thus, a bias to attend in the direction the trunk points would alert us to events at a long-term focus of attention while we temporarily look elsewhere.

To reveal potential trunk orientation biases in healthy individuals, we created experimental conditions under which a trunk orientation bias might be advantageous. Although data from patients with unilateral neglect support the hypothesis that the trunk is important to the distribution of spatial attention, studies of healthy individuals have produced mixed findings (Grubb \& Reed, 2002; Karnath, 1997; Karnath et al., 1991; Rorden et al., 2001). We hypothesized that these inconsistent results could be attributed to simple experimental tasks that did not have strong attentional demands and that did not require the trunk midline as a spatial anchor (i.e., tasks performed sitting in a chair). In such conditions, a trunk orientation bias would not have been necessary and might even have been detrimental to task performance.

In our study, we compared participants' performance on a lateralized target detection task while they were walking on a treadmill or standing still. Their heads were held at a $45^{\circ}$ angle relative to trunk midline, and targets were presented $45^{\circ}$ from fixation. Thus, one target was always in front of the trunk, and one was always to the side of one shoulder. In this setup, a trunk orientation bias was indicated by a speeded response to targets positioned in front of the trunk. In Experiment 1, we compared target detection performance during standing and walking conditions. To distinguish whether a trunk orientation bias was associated with trunk direction or the direction of motion, we not only had participants walk forward with their trunk midlines aligned with the direction of motion; we also had them walk sideways, so that their trunk midline was dissociated from their direction of motion. We found a trunk orientation bias in both walking conditions, whether or not the trunk was aligned with the direction of motion: When walking, but not when standing still, participants responded significantly more quickly to targets that appeared in front of their trunks than to targets that appeared off to the side. This finding supports theories that trunk orientation is an important anchor for spatial attention. Furthermore, the attentional bias was genuinely related to trunk orientation and not to the direction of simulated motion.

In Experiment 2, we explored how walking contributed to trunk-biased processing by manipulating motor and/or attention demands. Participants walked at four different speeds: $0,2.41,3.70$, and $4.83 \mathrm{~km} / \mathrm{h}$. We reasoned that if the trunk orientation bias were related to motor activity, it would be strongest at the fastest walking speed, but that if it were related to the attention demands produced by walking slower than a typical pace, it would be strongest at the slow walking speed. We found that, in addition to replicating the trunk orientation bias for walking conditions found in Experiment 1, the magnitude of this trunk orientation bias differed with walking speeds. Participants demonstrated significant trunk orientation biases only when walking at an unusually slow pace. This pattern of results appeared to stem from the greater motor and/or at- tention demands that participants reported were necessary to match the slow speed of the treadmill, rather than the increased motor activity per se required at faster walking speeds. Although we did not objectively measure arousal, it is likely that participants' arousal increased with walking speed, which speeded overall RTs.

To discriminate between motor and attention effects, in Experiment 3 we pitted performance with increased motor demands against increased cognitive demands in a dualtask paradigm. Motor load was assessed by comparing walking and standing conditions. Cognitive load was assessed by comparing concurrent counting conditions with nonconcurrent counting conditions. We found that motor load contributed more to the trunk orientation biases than did cognitive load; motor load by itself produced relatively faster responses in the direction of the trunk. Further, as in the other experiments, motor activity tended to speed RTs in general. We did find that cognitive load slowed responses but did not interact with trunk orientation.

These results have three important implications. First, trunk orientation is an anchor for spatial attention in healthy participants. When trunk orientation was dissociated from the simulated direction of motion, participants' attention continued to be biased toward trunk orientation. This suggests that the trunk orientation has some intrinsic attentional value, especially when it is relevant to the task. By extension, these results also indicate that trunk orientation effects on attention are not merely artifacts of unilateral neglect.

Second, although there is a trunk orientation bias, it is only present — or at least behaviorally manifested - in specific situations. Previous studies have produced mixed findings of trunk orientation biases in participants performing spatial attention tasks. Consequently, it was not clear whether trunk orientation biases were specific to certain groups of participants, to certain experimental tasks, or to a combination of the two. In this study, we were able to reliably modulate the behavioral presence or absence of the trunk orientation bias within subjects, and as measured by a single attention task. It appears that a trunk orientation bias is present under conditions in which the trunk is relevant to the task (i.e., when one is walking).

Third, the strength of the trunk orientation bias appears to be related to the effects of motor load on the attention system, and is not a general effect of arousal or cognitive load. An arousal explanation account for trunk orientation bias effects cannot explain the appearance of the bias at a slow walking speed. Furthermore, we can discount a purely cognitive explanation for trunk orientation bias, because increased cognitive load only had a general effect on RTs and was not related to trunk orientation. It is only when motor load is increased that a trunk orientation bias emerges.

The results of the present study emphasize the importance of the human body and its actions to visual attention. They demonstrate that a trunk orientation bias exists under behaviorally significant conditions and, in particular, when attention is required by motor activity. In conclusion, this study emphasizes the embodied nature of attention. When spatial attention is studied during locomotion 
and other tasks requiring human action, contributions from the trunk must be taken into consideration.

\section{AUTHOR NOTE}

This research was supported by NINDS F31 NS42873-02 and a University of Denver Partners-in-Scholarship grant. Correspondence concerning this article should be addressed to C. L. Reed, Department of Psychology, Claremont McKenna College, 850 Columbia Ave., Claremont, CA 91711 (e-mail: cathy.reed@cmc.edu).

\section{REFERENCES}

Biguer, B., Jeannerod, M., \& Prablanc, C. (1985). The role of position of gaze in movement accuracy. In M. I. Posner \& O. S. M. Marin (Eds.), Attention and performance XI (pp. 407-424). Hillsdale, NJ: Erlbaum.

Bradshaw, J. L., \& Mattingley, J. B. (1995). Clinical neuropsychology. New York: Academic Press.

BraIN, W. R. (1941). Visual disorientation with special reference to lesions of the right cerebral hemisphere. Brain, 64, 224-272.

BRIDGER, R. S. (1995). Introduction to ergonomics. New York: McGrawHill.

Broadbent, D. E. (1958). Perception and communication. London: Pergamon.

Deutsch, J. A., \& Deutsch, D. (1963). Attention: Some theoretical considerations. Psychological Review, 70, 80-90.

Diedrich, F. J., \& WARren, W. H. (1995). Why change gaits? The dynamics of the walk-run transition. Journal of Experimental Psychology: Human Perception \& Performance, 21, 181-202.

GrubB, J. D., \& ReED, C. L. (2002). Trunk orientation induces neglectlike lateral biases in covert attention. Psychological Science, 13, 553-556

Hasselbach-Heitzeg, M. M., \& Reuter-Lorenz, P. A. (2002). Egocentric body-centered coordinates modulate visuomotor performance. Neuropsychologia, 40, 1822-1833.

James, W. (1948). Psychology. Cleveland: Fine Editions Press.

Jeannerod, M., \& Biguer, B. (1987). The directional coding of reaching movements: A visuomotor conception of spatial neglect. In M. Jeannerod (Ed.), Neurophysiological and neuropsychological aspects of spatial neglect (pp. 87-113). Amsterdam: Elsevier.

JimeneZ, L., \& VAZqueZ, G. A. (2005). Sequence learning under dualtask conditions: Alternatives to a resource-based account. Psychological Research, 69, 352-368.

Kahneman, D. (1973). Attention and effort. Englewood Cliffs, NJ: Prentice Hall.

Kahneman, D., \& Treisman, A. (1984). Changing views of attention and automaticity. In R. Parasuraman \& D. R. Davies (Eds.), Varieties of attention (pp. 29-61). Orlando: Academic Press.

KaRNATH, H.-O. (1994). Subjective body orientation in neglect and the interactive contribution of neck muscle proprioception and vestibular stimulation. Brain, 117, 1001-1012.

KARNATH, H.-O. (1997). Neural encoding of space in egocentric coordinates? Evidence for and limits of a hypothesis derived from patients with parietal lesions and neglect. In P. Thier \& H.-O. Karnath (Eds.), Parietal lobe contributions to orientation in $3 D$ space (pp. 497-520). Berlin: Springer.

Karnath, H.-O., Christ, K., \& HartJe, W. (1993). Decrease of contralateral neglect by neck muscle vibration and spatial orientation of trunk midline. Brain, 116, 383-396.

Karnath, H.-O., Schenkel, P., \& Fischer, B. (1991). Trunk orientation as the determining factor in the "contralateral" deficit in the neglect syndrome and as the anchor of the internal representation of the body in space. Brain, 114, 1997-2014.

National AERonautics and SPaCe Administration Glenn Research CENTER (2002). NASA Glenn safety manual: $\mathrm{CH}$-30 ergonomics. Available at osat-ext.grc.nasa.gov/gso/manual/chapter_30.pdf.

Oregon OCCUPATIONAL SAFETy AND Health Administration (2002). Health and safety guidelines for video display terminals in the workplace (OR-OSHA Publication No. 440-1863 [12/02]). Salem, OR: Author.

Pizzamiglio, L., Frasca, R., Guariglia, C., Incoccia, C., \& AntoNUCCI, G. (1990). Effect of optokinetic stimulation in patients with visual neglect. Cortex, 26, 535-540.

Previc, F. H. (1998). The neuropsychology of 3-D space. Psychological Bulletin, 124, 123-164.

PRINZ, W. (1997). Perception and action planning. European Journal of Cognitive Psychology, 9, 129-154.

RoRDEN, C., KARNATH, H.-O., \& DrIVER, J. (2001). Do neck-proprioceptive and caloric-vestibular stimulation influence covert visual attention in normals, as they influence visual neglect? Neuropsychologia, 39, 364-375.

RUBENS, A. B. (1985). Caloric stimulation and unilateral visual neglect. Neurology, 35, 1019-1024.

Vallar, G., Guariglia, C., \& Rusconi, L. (1997). Modulation of the neglect syndrome by sensory stimulation. In P. Thier \& H.-O. Karnath (Eds.), Parietal lobe contributions to orientation in $3 D$ space (pp. 556-578). Berlin: Springer.

Ventre, J., Flandrin, J. M., \& Jeannerod, M. (1984). In search for the egocentric reference: A neurophysiological hypothesis. Neuropsychologia, 22, 797-806.

(Manuscript received September 3, 2004; revision accepted for publication September 16, 2007.) 